\title{
Location of the Flexor Carpi Radialis Myotendinous Junction: a Cadaveric Study
}

\author{
K. Panwar, D. Lara, M. Trzeciak, E. G. Huish Jr \\ Valley Orthopedic Surgery Residency, Modesto (CA), U.S.A.
}

\section{CORRESPONDING AUTHOR:}

Kunal Panwar

Valley Orthopedic Surgery Residency

3055 Floyd Avenue

Modesto (CA) 95355, U.S.A.

E-mail: kpanwar123@gmail.com

DOI:

10.32098/mltj.04.2021.20

LEVEL OF EVIDENCE: 5

\begin{abstract}
SUMMARY
Background. The flexor carpi radialis (FCR) tendon is frequently selected for use as an interposition graft, for tendon transfers, and as a landmark for volar forearm approaches. Original surgical techniques of FCR harvest recommend incision placement $10 \mathrm{~cm}$ proximal to wrist crease. To date no anatomic study has been conducted to precisely define the location of the FCR myotendinous junction.

Methods. 5 fresh frozen cadavers were dissected, exposing the full length of the FCR. The radial styloid (RS) was selected as a distal anatomic landmark, the medial epicondyle $(\mathrm{ME})$ was chosen as a proximal landmark. All measurements were taken along the length of the FCR tendon. As the FCR myotendinous junction is chevron shaped, we marked both the proximal myotendinous junction (PMT) and distal myotendinous junction (DMT), with the DMT indicating the beginning of purely tendinous FCR. Four measurements were taken for each arm: 1) RS to DMT, 2) RS to PMT, 3) ME to PMT, 4) ME to DMT.

Results. Pearson correlation coefficient comparing tendinous length of the FCR to the overall forearm length was 0.896 indicating a strong positive correlation $(\mathrm{p}=0.040)$. The mean ratio of tendinous FCR length to forearm length was $0.42 \pm 0.05$, ranging from 0.38 to 0.50 . This correlated to a distance $11.7 \pm 2.3 \mathrm{~cm}$ proximal from the radial styloid.

Conclusions. This study demonstrates the location of the myotendinous junction of the FCR. Our results suggest incision placement $10 \mathrm{~cm}$ from proximal wrist crease to be unreliable in forearms too short or too long. Instead, we recommend measuring $40 \%$ of the length from RS to ME as a more consistent marker for FCR harvest.
\end{abstract}

\section{KEY WORDS}

Flexor Carpi Radialis; cadaveric study; myotendinous junction; tendon harvest; volar forearm anatomy.

\section{INTRODUCTION}

The flexor carpi radialis (FCR) is a wrist flexor and radial deviator found in the superficial compartment of the volar forearm. It originates at the common flexor origin on the medial epicondyle and inserts primarily at the base of the second metacarpal. It is a frequently selected tendon for use in carpometacarpal (CMC) arthroplasty interposition, tendon transfer, and as a landmark for volar forearm approaches $(1-6,10)$. To date no anatomic study has been conducted to delineate the precise location of the FCR myotendinous junction. We hypothesized that the FCR myotendinous junction has a predictable anatomic relationship to the radial styloid (RS) and medial epicondyle (ME).

\section{MATERIALS AND METHODS}

In accordance with established ethical standards, 5 fresh frozen cadavers were selected involving fully preserved upper extremities (Padulo et al. (11)). All cadavers were placed in full supination, 90 degrees of elbow flexion, and 
secured to a table. Skin was carefully dissected out over the volar forearm exposing the full length of the FCR. The RS was selected as a distal anatomic landmark, and a line was drawn across the volar wrist perpendicular to it. Similarly, the $\mathrm{ME}$ was marked as a proximal landmark. All measurements were taken as a straight line along the length of the FCR tendon. Since the FCR myotendinous junction is chevron shaped, both the proximal myotendinous junction (PMT) and distal myotendinous junction (DMT) were demarcated. Four measurements were taken for each arm: 1) RS to DMT, 2) DMT to ME, 3) RS to PMT, 4) PMT to ME. In the interest of highlighting clinical relevance, the DMT indicates the location of purely tendinous FCR (figure 1).

\section{Statistical methods}

Statistical analysis was performed with SPSS version 25 (IBM, Armonk, NY). Pearson correlation was performed to compare the forearm length (measured from the tip of the radial styloid to medial epicondyle) with the tendinous length of the FCR (measured from the insertion to the distal most aspect of the musculotendinous junction). Descriptive statistics were then calculated.

\section{RESULTS}

The distances measured in each cadaveric specimen are shown in table I. Pearson correlation coefficient comparing tendinous length of the FCR to the overall forearm length was 0.896 indicating a strong positive correlation $(\mathrm{p}$ $=0.040$ ). The mean ratio of tendinous FCR length to forearm length was $0.42 \pm 0.05$, ranging from 0.38 to 0.50 . This correlated to a distance $11.7 \pm 2.3 \mathrm{~cm}$ proximal from the radial styloid.

\section{DISCUSSION}

Since the original description of ligament reconstruction and tendon interposition (LRTI) for CMC arthritis by Eaton and Littler in 1973, various techniques have been described

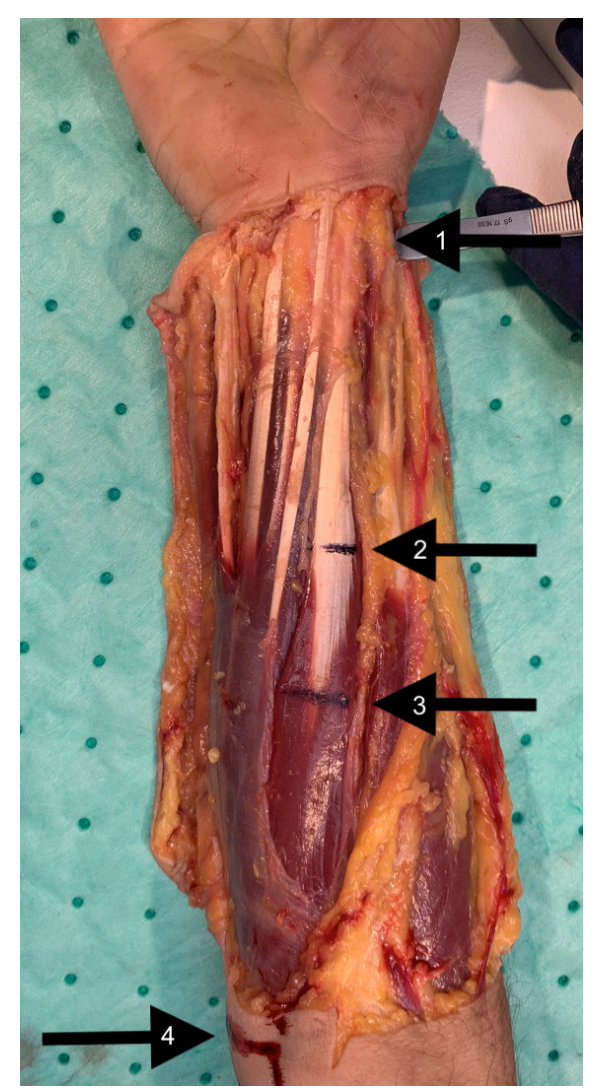

Figure 1. Volar Forearm Exposure of the Flexor Carpi Radialis Tendon.

Arrow 1 indicates the radial styloid, arrow 2 indicates the distal most portion of the musculotendinous junction, arrow 3 indicates the proximal most portion of the musculotendinous junction, arrow 4 indicates the medial epicondyle.

for FCR harvest (2, 7-9). Some of these techniques require at least four separate incisions, with the proximal most extent of the FCR tendon estimated to be $10 \mathrm{~cm}$ from the proximal wrist crease. This, however, fails to consider patients of different sizes and forearm lengths. One specimen showed the distal end of the myotendinous junction to be only 9.5 $\mathrm{cm}$ from the radial styloid, which would be more distal than the oft-estimated $10 \mathrm{~cm}$. Also, many of the specimens

Table I. Measurements taken from each cadaveric specimen.

\begin{tabular}{llllll}
\hline Specimen & RS to DMT $(\mathbf{C M})$ & DMT to ME $(\mathbf{C M})$ & RS to PMT (CM) & $\begin{array}{l}\text { PMT } \\
\text { to ME }(\mathbf{C M})\end{array}$ & Total Length $(\mathrm{CM})$ \\
\hline 1 & 14.5 & 14.5 & 19.0 & 10.0 & 29.0 \\
\hline 2 & 9.5 & 15.2 & 13.2 & 11.5 & 24.7 \\
\hline 3 & 10.3 & 17.1 & 15.6 & 11.8 & 27.4 \\
\hline 4 & 13.8 & 16.3 & 17.8 & 12.3 & 30.1 \\
\hline 5 & 10.5 & 16.2 & 13.7 & 12.9 & 26.6 \\
\hline
\end{tabular}

RS: radial styloid; ME: medial epicondyle; DMT: distal myotendinous junction; PMT: proximal myotendinous junction. 
showed the distal end of the myotendinous junction to be much further including the largest measuring $14.5 \mathrm{~cm}$. Our results demonstrate that the tendinous portion of the FCR is located $11.7 \pm 2.3 \mathrm{~cm}$ proximal from the radial styloid. This correlates to $42 \%$ of the distance from radial styloid to medial epicondyle. As such, conventional descriptions of incision placement $10 \mathrm{~cm}$ from proximal wrist crease would be sufficient for most indications, but for patients with very short or very long forearms, adjustments should be made.

\section{CONCLUSIONS}

The primary limitation of the study is its sample size of 5 cadavers; however, the strong correlation found between forearm

\section{REFERENCES}

1. Burton RI, Pellegrini VD Jr. Surgical management of basal joint arthritis of the thumb. Part II. Ligament reconstruction with tendon interposition arthroplasty. J Hand Surg Am 1986;11(3):324-32.

2. Eaton RG, Littler JW. Ligament reconstruction for the painful thumb carpometacarpal joint. J Bone Joint Surg Am 1973;55(8):1655-66.

3. Vermeulen GM, Brink SM, Sluiter J, Elias SG, Hovius SE, Moojen TM. Ligament reconstruction arthroplasty for primary thumb carpometacarpal osteoarthritis (weilby technique): prospective cohort study. J Hand Surg Am 2009;34(8):1393-401.

4. Daniel C. Riordan. Tendon transfers for median, ulnar or radial nerve palsy. Hand 1969;1(1):42-6.

5. Riordan DC. Tendon transfers in hand surgery. J Hand Surg Am 1983;8(5 Pt 2):748-53.

6. Karabeg R. Assessment of the Forearm Tendon Transfer with Irreparable Radial Nerve Injuries Caused by War Projectiles. Med Arch 2019;73(6):415-20. length and FCR tendon length reached statistical significance. Larger sample size cadaveric studies would prove beneficial toward further evaluation of FCR tendinous anatomy. The authors acknowledge a further limitation in that elbow flexion was controlled at 90 degrees during all measurements because one of the cadavers did not have full elbow extension. This could create a slight disparity between cadaveric measurements (taken in elbow flexion) and intra-operative measurements (performed in full extension).

\section{CONFLICT OF INTERESTS}

The authors declare that they have no conflict of interests.

7. Umarji S, Pickford M. Re: a novel technique for harvesting a split flexor carpi radialis (FCR) tendon graft. J Hand Surg Eur Vol 2008;33(6):817-8.

8. Tomaino MM, Pellegrini VD Jr, Burton RI. Arthroplasty of the basal joint of the thumb. Long-term follow-up after ligament reconstruction with tendon interposition. J Bone Joint Surg Am 1995;77(3):346-55.

9. Lester B, McCormack RR Jr, Jeong GK. The wire interlock technique for harvesting a partial-width distally attached tendon graft. J Hand Surg Am 2000;25(1):176-82.

10. Igeta Y, Vernet P, Facca S, et al. The minimally invasive flexor carpi radialis approach: a new perspective for distal radius fractures. Eur J Orthop Surg Traumatol 2018;28(8):1515-1522. Erratum in: Eur J Orthop Surg Traumatol 2018 Mar 3; Diaz JJH [corrected to Hidalgo Diaz JJ].

11. Padulo J, Oliva F, Frizziero A, Maffulli N. Muscles, Ligaments and Tendons Journal - Basic principles and recommendations in clinical and field Science Research: 2018 update. Muscles Ligaments Tendons J 2018;8(3):305-7. 